\title{
Defining fitness for purpose in South African anaesthesiologists using a Delphi technique to assess the CanMEDS framework
}

\author{
Kalafatis N* (D), Sommerville T, Gopalan P \\ Department of Anesthesiology and Critical care, Nelson R Mandela School of Medicine, University of KwaZulu Natal, Durban, South Africa \\ *Corresponding author, email: nickykalafatis@gmail.com
}

Background: Training of South African anaesthesiologists is based on the Canadian Medical Education Directives for Specialists (CanMEDS). However, the applicability of CanMEDS in this context has not been assessed. An expert panel participated in a Delphi process to create an appropriate expanded list of CanMEDS competencies that may be used in the future to assess fitness for purpose of local graduates.

Methods: This descriptive study comprised a representative panel of 16 experts surveyed electronically over three rounds to assess the importance of the existing CanMEDS roles and enabling competencies and suggested additions deemed applicable locally. The primary outcome was the creation of a list of competencies applicable to South Africa.

Results: There was a $100 \%$ response rate for all three rounds. Based on the existing seven CanMEDS meta-competencies (Medical Expert, Collaborator, Communicator, Leader, Scholar, Professional and Health Advocate), respondents scored the importance of 89 enabling competencies and 19 additional competencies. Seven CanMEDS enabling competencies did not achieve consensus and were excluded. Nineteen new enabling competencies and two new meta-competencies (Humaneness, Context Awareness) achieved consensus and were added. Median ratings of importance of meta-competencies showed highest scores for Medical Expert and Collaborator and lowest scores for Health Advocate. Weighting of meta-competencies revealed highest scores for Medical Expert and Professional with all others equally weighted.

Conclusion: This study has formulated an adapted CanMEDS list of enabling competencies with the addition of the two new metacompetencies of Context Awareness and Humaneness for use in South African anaesthesiology. This provides a means with which future graduates may be assessed for fitness for purpose.

Keywords: fitness for purpose, anaesthesiology, medical education

\section{Introduction}

In South Africa, anaesthesiologists are a scarce commodity with an estimated 2.89 per 100000 population. (South African Society of Anaesthesiology, personal communication). Due to an everincreasing demand, local anaesthesiologists do not necessarily remain in the public sector to evolve into their specialist roles. As in other countries, they have to function independently irrespective of their placement immediately postgraduation to address the large demand placed on the healthcare sector. The transition from trainee to specialist has been associated with adverse physician and patient outcomes, primarily due to the perception of newly qualified specialists of unpreparedness. ${ }^{1-5}$ To alleviate this additional stressor, graduates need to be fit for purpose to function safely and appropriately.

Fitness for purpose (FFP) in anaesthesiology has been defined as an expert without any deficiencies in either their technical or nontechnical skills. ${ }^{6}$ It has, however, not been addressed in the South African anaesthesiology context. This elusive concept is closely related to competence and expertise, however, it is imperative that distinctions are made to have a clear understanding of the expectations of graduating specialist anaesthesiologists. ${ }^{6}$

In South Africa, anaesthesiologists under the guidance of the Colleges of Medicine of South Africa (CMSA) are trained by university departments and assessed in accordance with the Canadian Medical Education Directives for Specialists (CanMEDS).
CanMEDS was designed by The Royal College of Physicians and Surgeons in Canada in $2005^{7}$ and has been recently updated ${ }^{8}$ (Figure 1). The CanMEDS competency framework guides and informs the teaching and training of medical specialists in order to achieve competence in their speciality whilst simultaneously ensuring societal accountability. ${ }^{7}$

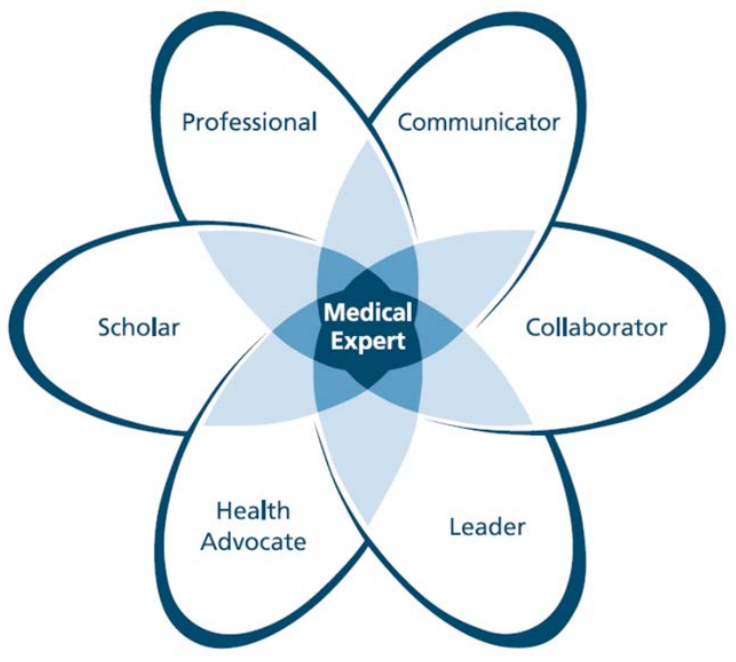

\section{$\underset{\text { OF PHYSICANS AND SURGEOLS OFGE GANADA }}{\text { ROYALNM }}$ C D D}

Figure 1. The CanMEDS (Canadian Medical Education Directives for Specialists) Roles. Copyright $\odot 2015$ The Royal College of Physicians and Surgeons of Canada. http://rcpsc.medical.org/canmeds. (Reproduced with permission) 
In Canada, CanMEDS evolved according to societal needs and stakeholders were intimately involved in the design and planning of the framework with specific competency outcomes in mind. ${ }^{9}$ However, other countries adopting CanMEDS have not necessarily gone through the same rigorous processes for their specific needs. This framework may not be inclusive enough for the distinctiveness of specific specialities and requires validation for anaesthesiology in the South African context.

Several studies have investigated the appropriateness of the adoption of CanMEDS for local specialist training. A Danish study assessed the validity of CanMEDS for the training of all specialities in Denmark. ${ }^{9}$ This study confirmed that despite considering CanMEDS mostly valid, it required internal validation for specific specialities, local societal needs and variations in local medical education and patient care. A study conducted in Germany adapted the seven CanMEDS roles for an anaesthesiology department by taking into account the specific needs of anaesthesia in that setting. ${ }^{10}$

The current South African anaesthesiology curriculum comprises 13 domains rooted in the existing seven CanMEDS generic meta-competencies. Several potential problems with CanMEDS exist. Firstly, it is a competency framework that, despite its many benefits to postgraduate training and improved patient outcomes, does not imply fitness for purpose. ${ }^{6}$ Each of the seven meta-competencies consists of several key and enabling competencies incorporated into milestones that the graduate must achieve. ${ }^{11}$ However, not all meta-competencies or complex roles can be reduced to component milestones. Assuming that a graduate can perform a complex task based on their ability to perform the components of the task is not necessarily true. ${ }^{12-14}$
Secondly, several deficiencies have been demonstrated in nontechnical components of the CanMEDS framework because they are more difficult to teach and therefore assess, ${ }^{15}$ rendering graduates deficient in these skills. ${ }^{16}$ The competence suggested by CanMEDS cannot assure fitness for purpose since not all nontechnical skills are incorporated.

\section{Aim of the study}

The study's purpose was to determine whether CanMEDS was an appropriate framework for FFP in South African anaesthesiology as determined by local experts. Consideration was specific to what core roles and enabling competencies every anaesthesiologist in South Africa should possess, irrespective of their eventual placement in either the public or private sectors. This would assist in the ultimate creation of an appropriate list of criteria to assess fitness for purpose.

\section{Methods}

\section{Ethics}

This study received ethical approval from the Biomedical Research Ethics Committee (BREC) at the University of KwaZulu Natal (BE199/17). All participants provided informed consent.

\section{Study details}

A qualitative Delphi-designed study consisting of three electronic survey rounds (Survey Monkey) was undertaken. The study sample comprised South African anaesthesiologists deemed to be experts in the field, representative of both the public and private sectors, and totalled sixteen. Half represented

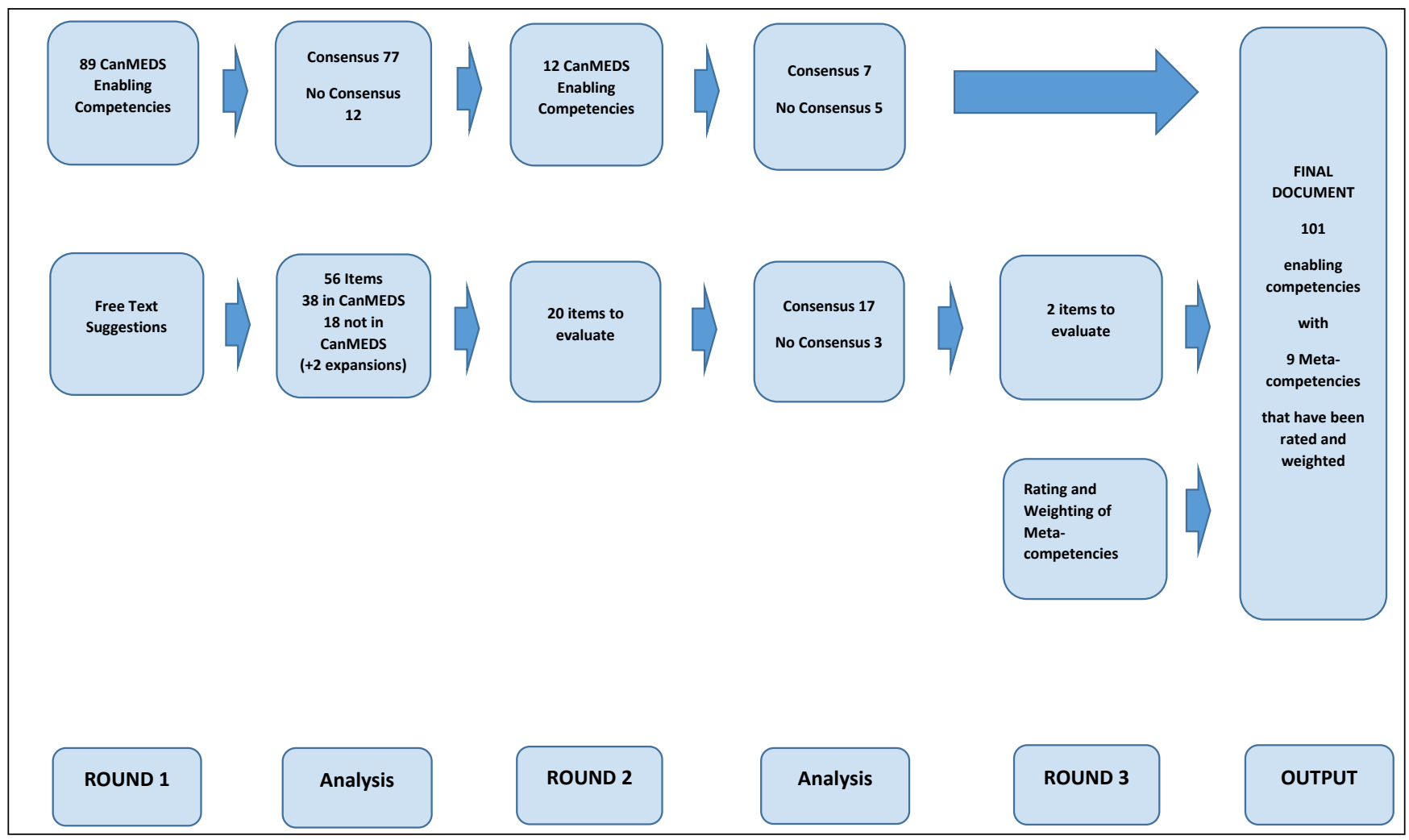

Figure 2. Overview of the multi-phase Delphi process 
the public sector, comprising heads of the eight departments of anaesthesiology affiliated to the eight national medical schools. The other half represented the private sector in the geographical distribution of each academic department and were involved in teaching and training in their affiliated departments. All respondents remained anonymous to the other participants throughout the Delphi process.

An a priori consensus level was determined as $80 \%(\mathrm{~N}=13)$ for scoring using a 4-point Likert scale (1 - not important at all; 2 - somewhat important; 3 - very important; 4 - absolutely essential). The investigators stipulated a two-round scoring process for each item. If consensus was not obtained after two rounds, the item was excluded.

In round one, participants scored the importance of enabling competencies of the seven existing CanMEDS roles in the context of South African anaesthesiologists working at any level in both the private and public sectors. Scores of 1 and 2 were considered unworthy of inclusion with items thus excluded, while scores of 3 and 4 were worthy of inclusion and thus retained. At the end of round one, respondents suggested any enabling competencies or new roles appropriate for South African anaesthesiology that had not been incorporated into the existing CanMEDS framework.

Round two required rescoring of items for which consensus had not been achieved in round one and scoring of the suggested additions made by respondents in round one. Round three required rescoring of the additional items for which consensus had not been reached in the previous round and also allowed respondents to rate and weight the importance of the various meta-competencies considered appropriate in the local context. Prior to the commencement of rounds two and three, respondents received feedback in the form of their score in relation to the median score of the panel from the preceding round.

The primary outcome was the creation of a consensus list of core meta-competencies and enabling competencies that South African anaesthesiologists would be expected to possess on graduation that would contribute to their fitness for purpose. These competencies would provide a means with which to both define and measure fitness for purpose in the South African anaesthesiology context (Figure 2).

\section{Results}

Sixteen participants were identified, with all agreeing to participate in the study. The final panel consisted of eight public and eight private sector anaesthesiologists affiliated to academia, deemed to be considered local experts and representative of their geographical and training centre location. There was a $100 \%$ response rate for all three rounds and all questions were answered.

\section{Round 1}

Round one comprised two components: a) scoring of existing CanMEDS competencies and b) free text to allow for respondent suggestions. Ninety-seven questions were included with 89 representing the enabling competencies of the seven existing CanMEDS specialist roles that required scoring. Eight questions prompted respondents to consider whether any new roles or enabling competencies for existing roles were absent from the current CanMEDS framework (free text). At the end of round one, consensus was achieved for 77 of the 89 enabling competencies, all with scores of 3 or 4 (worthy of inclusion). Non-consensus competencies proceeded to round two (Table I).

Table I. Summary of CanMEDS enabling competency scoring for rounds one to three.

\begin{tabular}{|c|c|c|c|c|c|c|c|c|}
\hline & 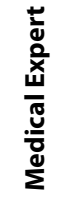 & 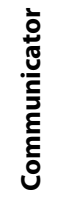 & 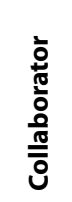 & 离 & 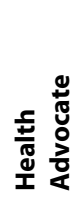 & 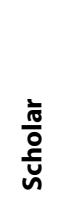 & 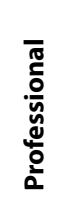 & 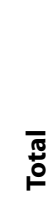 \\
\hline \multicolumn{9}{|l|}{ ROUND 1} \\
\hline Enabling competencies & 17 & 17 & 7 & 11 & 6 & 18 & 13 & 89 \\
\hline Consensus achieved & 17 & 16 & 7 & 10 & 0 & 15 & 12 & 77 \\
\hline \multicolumn{9}{|l|}{ ROUND 2} \\
\hline $\begin{array}{l}\text { Enabling competencies } \\
\text { (carried over from R1) }\end{array}$ & 0 & 1 & 0 & 1 & 6 & 3 & 1 & 12 \\
\hline Consensus achieved & N/A & 0 & N/A & 1 & 3 & 1 & 0 & 5 \\
\hline $\begin{array}{l}\text { Enabling competencies } \\
\text { Deleted after R2 }\end{array}$ & 0 & 1 & 0 & 0 & 3 & 2 & 1 & 7 \\
\hline \multicolumn{9}{|l|}{ ROUND 3} \\
\hline Enabling competencies & N/A & N/A & $\mathrm{N} / \mathrm{A}$ & $\mathrm{N} / \mathrm{A}$ & $\mathrm{N} / \mathrm{A}$ & N/A & N/A & $\mathrm{N} / \mathrm{A}$ \\
\hline
\end{tabular}

In the second (free text) component of round one, respondents considered what enabling competencies and /or roles were omitted from or insufficiently represented by the existing generic CanMEDS framework deemed necessary for South African anaesthesiology. Respondents provided 56 additional suggestions. These were categorised into related themes and cross-referenced with the CanMEDS 2015 framework $^{8}$ to avoid duplication. Of these, eighteen either did not form part of the existing CanMEDS framework or were considered inappropriately represented in the framework for the local context (Table II). These were retained for consideration in round two (Table III).

Of the proposed 18 additions for inclusion into round two, $44 \%$ were incorporated into existing CanMEDS roles with the remaining $10(56 \%)$ considered under two overarching new themes:

Humaneness, defined as tenderness, compassion, and sympathy for people, especially for those who suffer or are distressed, that causes the least harm to people and should underpin ALL the roles of a specialist; and 
Context awareness, defined as the global ability of a specialist to be aware of and consider how various clinical and non-clinical contexts would require a modification in practice to ensure good outcomes. This would include both situational and contextspecific adaptation that will permeate across ALL the roles of the specialist.

Table II. Summary of free text suggestions for additions to existing CanMEDS (rounds one to three)

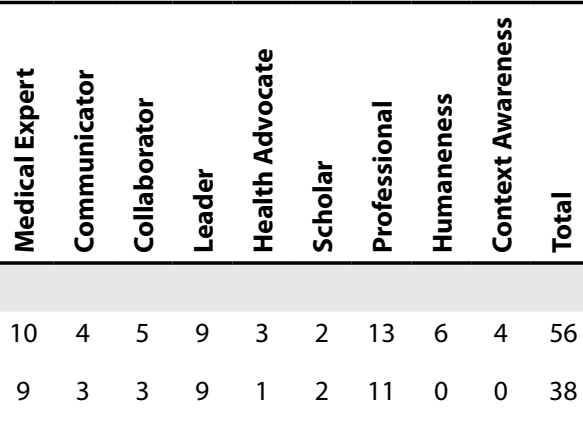

\begin{tabular}{|c|c|c|c|c|c|c|c|c|c|}
\hline Suggestions & 10 & 4 & 5 & 9 & 3 & 2 & 13 & 6 & 4 \\
\hline $\begin{array}{l}\text { Cross referenced } \\
\text { to CanMEDS }\end{array}$ & 9 & 3 & 3 & 9 & 1 & 2 & 11 & 0 & 0 \\
\hline
\end{tabular}

ROUND 2

Enabling

competencies

scored (carried

over from R1)

Consensus

achieved

$\begin{array}{lllllllll}1 & 1 & 2 & 0 & 2 & 0 & 3^{*} & 6 & 4\end{array}$

19

\section{ROUND 3}

Enabling

competencies

Consensus

achieved

$\begin{array}{lllllllll}\text { N/A N/A N/A N/A } & 1 & \text { N/A } & 1 & \text { N/A } & \text { N/A } & 2\end{array}$

R1 - round one; R2 - round two; R3 - round three; N/A - not applicable *One question carried over from R1 was considered as two separate questions in R2

\section{Round 2}

Round two comprised two components. The first consisted of the 12 remaining questions carried over from round one that required rescoring to reach consensus. The second section comprised 20 questions in total; 19 formulated from the 18 free text suggestions and one assessed relative importance of suggestions from round one.

The twelve enabling competencies carried over from round one were rescored with seven not reaching consensus and thus excluded (Table IV). The remaining five items all scored 3 or 4 and were thus considered worthy of inclusion (Table I).

Table IV. CanMEDS enabling competency deletions after rounds one and two

\begin{tabular}{|c|c|}
\hline Role & Enabling Competency Deleted \\
\hline Communicator & $\begin{array}{l}\text { Assist patients and their families to identify, } \\
\text { access, and make use of information and } \\
\text { communication technologies to support their } \\
\text { care and manage their health. }\end{array}$ \\
\hline Health Advocate & $\begin{array}{l}\text { Work with patients to address determinants } \\
\text { of health that affect them and their access to } \\
\text { needed health services or resources. }\end{array}$ \\
\hline Health Advocate & $\begin{array}{l}\text { Work with patients and their families to increase } \\
\text { opportunities to adopt healthy behaviours. }\end{array}$ \\
\hline Health Advocate & $\begin{array}{l}\text { Work with a community or population to identify } \\
\text { the determinants of health that affect them. }\end{array}$ \\
\hline Scholar & Contribute to the work of a research programme. \\
\hline Scholar & $\begin{array}{l}\text { Pose questions amenable to scholarly inquiry and } \\
\text { select appropriate methods to address them. }\end{array}$ \\
\hline Professional & $\begin{array}{l}\text { Participate in peer assessment and standard- } \\
\text { setting. }\end{array}$ \\
\hline
\end{tabular}

Table III. Summary of categorised round one free text suggestions for consideration in round two

Suggestion not part of/insufficiently represented by CanMEDS

Theme allocated to suggestion

Ability to multi-task. Medical Expert

The importance of a translator where there is a language barrier: effective communication to ensure patient understanding. Ability to decide on the appropriate level of competence of the healthcare provider to whom patient care is handed over (e.g. awareness of level of competency required at handover).

Ability to respond to need for involvement outside perioperative domain e.g. mass casualties. Communication

Collaborator

Ensure that all staff (including oneself) in the clinical environment are safe and not at risk with respect to physical or

Collaborator psychological injuries.

In-depth knowledge of global health issues

Advocacy on behalf of the profession to the public: public opinion of the profession.

Health Advocate

Punctual and efficient practice.

Adaptability and creativity in various situations with respect to solving problems.

Health Advocate

Professional

Professional

Context Awareness

Ability to assess capacity to consent in various contexts: context specific adaptation.

Context awareness

Remain humane despite inhumane demands.

Context awareness

Situational awareness: the ability to perceive, comprehend the meaning of, and to predict the impact of variables in the work environment.

Tolerance of diversity/differences and able to practise non-judgementally.

Humaneness

Ability to remain calm under pressure.

Possess an awareness of and respect for the impact and sensitivities of race, culture, ethnicity and gender differences on decision-making and patient management.

Honesty, integrity, altruism, humility.

Humaneness

Humaneness

Humaneness

Manage patients and their families/caregivers with a caring and empathetic nature.

Humaneness

Humaneness 
In the second component, nineteen enabling competencies were scored for the first time. Two did not reach consensus and were forwarded to round three (Table II). All consensus scores were either 3 or 4 and were included. The final question in this component interrogated the relative importance of CanMEDS roles. Consensus was not achieved requiring rescoring in round three.

\section{Round 3}

Round three addressed three outstanding questions from round two. At the outset, respondents were asked to re-score two enabling competencies to reach consensus (Table II), which was achieved. Thereafter, a final modified CanMEDS list for South African anaesthesiology was created (Appendix).

To investigate the relative importance of CanMEDS roles, the third question was expanded to include all existing CanMEDS roles as well as the two additions of Humaneness and Context Awareness. The expanded questions required a) rating of importance of the roles using a 9-point scale and the b) weighting of all roles as a percentage of time spent for teaching, training and assessment (Figures 3 and 4).

\section{Discussion}

This descriptive study examined the views of experts in South African anaesthesiology to ascertain whether the existing CanMEDS competency framework is appropriate for establishing fitness for purpose in local anaesthesiologists. The results indicate that all seven CanMEDS roles and the majority (82/89) of the CanMEDS enabling competencies are considered important

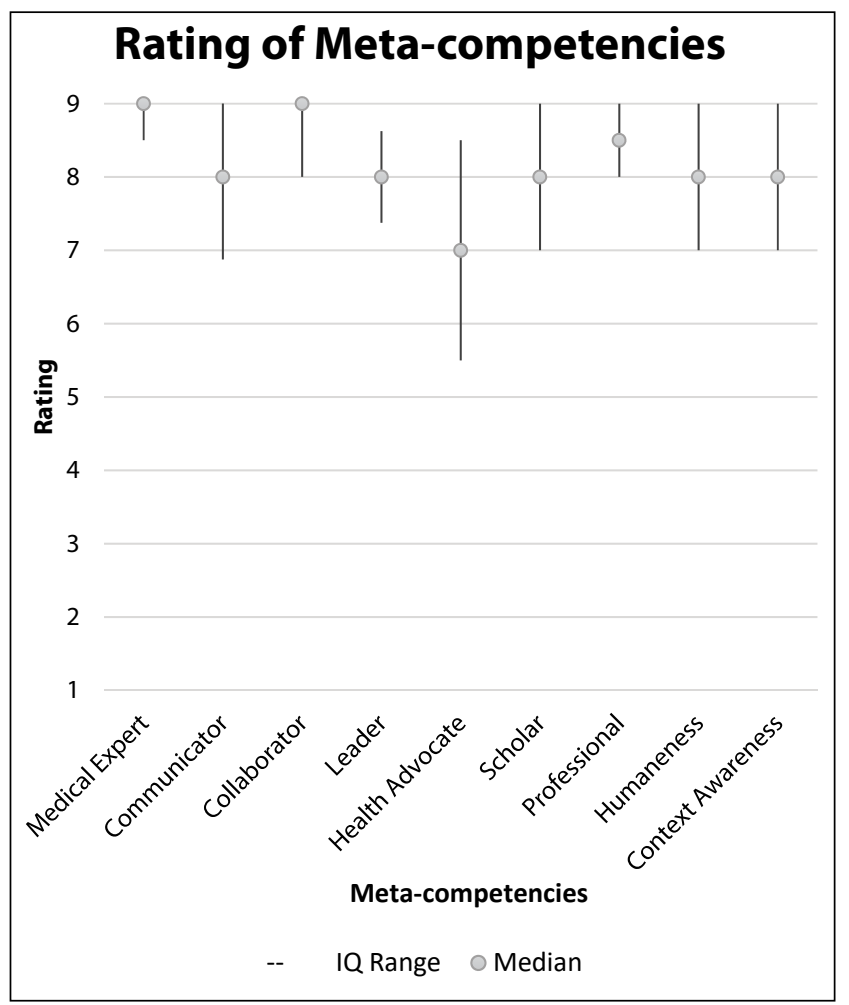

Figure 3. Round 3 median rating of importance of meta-competencies. Rankings done on 9-point scale with $1=$ LEAST important and $9=$ MOST important. locally. This attests to the applicability of this framework, albeit generic in nature, to most aspects of local anaesthetic practice. However, seven CanMEDS enabling competencies where deemed unworthy and omitted (Table IV). Of those, the majority were associated with the role of Health Advocate. This reflects international sentiments ${ }^{9,17}$ of specialists including anaesthesiologists, consistently rating this the least important of all specialist roles. Our panel rated the importance of all nine meta-competencies highly, scoring all between seven and nine, with the lowest rated being Health Advocate ${ }^{9,17}$ (Figure 2).

Reasons for the low ratings of health advocacy despite inclusion into specialist training frameworks are varied. Some suggest trainees are not taught advocacy or may only advocate at an individual rather than at a societal level.9,17 Others report that specialists rate health advocacy lowest, unlike general practitioners, suggesting that advocacy needs within a hospital are deferred to those involved in primary health care. ${ }^{9}$ Verma, Flynn and Seguin report that Canadian faculties find it difficult to formally teach and assess advocacy, suggesting its learning via the hidden curriculum through experience rather than by formal teaching. ${ }^{17}$ Hence, if the healthcare system lacks good role modelling, this component of learning might be omitted, causing deficiencies. Important considerations of conflict may arise where specialists need to be managers in situations of resource constraints yet are expected to promote health advocacy for all. ${ }^{17}$ Health advocacy may be perceived as additional work by busy anaesthesiologists who are working in demanding situations with high clinical loads. Time constraints, poor remuneration and feelings of overwhelming demands may unfortunately render health advocacy a lower priority despite the acceptance of the social responsibility for advocacy to all. ${ }^{17}$ Despite deletions of some societal health advocacyrelated enabling competencies, our panel suggested additions

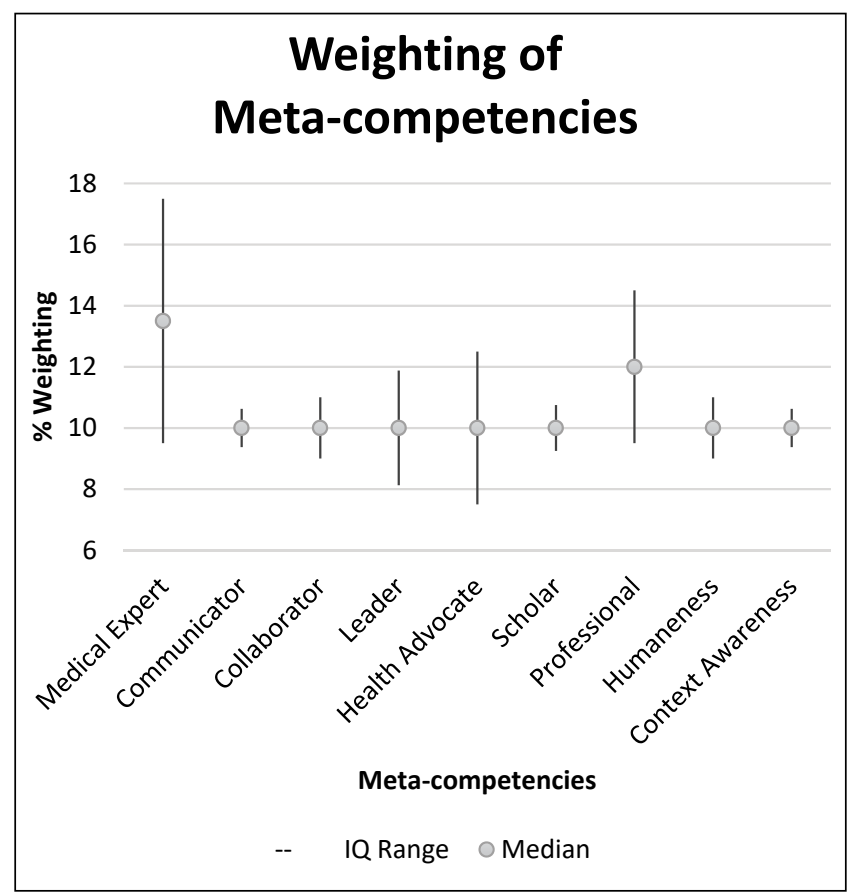

Figure 4. Round 3 median weighting of meta-competencies with respect to time to be spent on teaching, training and assessment. Median weighting reflected as a percentage (\%). 
more appropriate for the specialist anaesthesiologist, reflecting advocacy towards colleagues and self to ensure physician welfare (Table III).

Our panel rated the roles of Collaborator and Medical Expert highest. Results for Collaborator may suggest that anaesthesiologists appreciate that their expert perioperative management of patients cannot be performed in isolation and that collaboration with other disciplines is crucial to enhance patient outcomes. Collaboration is not only important for clinical care of patients but also with respect to training, teaching and research.

The high rating of Medical Expert reflects the perceived central role that this meta-competency plays in creating an expert. According to Dreyfus and Dreyfus, the trainee evolves from competent, to proficient and ultimately to an expert who has intuitive knowledge and can perform complex tasks. ${ }^{18}$ CanMEDS considers the Medical Expert as a physician who "integrates all of the CanMEDS roles, applying medical knowledge, clinical skills and professional values in their provision of high-quality and safe patient-centred care. It is the central physician role in the CanMEDS framework and defines the physician's clinical scope of practice".8 Although our panel echoes this sentiment it suggests that the core personal attributes considered important for practitioners to possess in order to be FFP are not entirely taken into account. Experts felt that the seven CanMEDS metacompetencies were not inclusive enough for the requirements of a local anaesthesiologist and suggested the addition of other features, categorised into roles of Humaneness and Context Awareness, to improve applicability in the local context.

Humaneness incorporates human attributes necessary to care for distressed or suffering individuals and describes the features of a good-natured individual of sound moral standards who responds appropriately to those in need. CanMEDS incorporates some aspects of Humaneness currently (altruism, humility, honesty, integrity ${ }^{8}$ but these have been isolated to the role of professional. The panel considered these attributes not only important in one's professional role but in every role of a specialist.

Due to the country's economic instability and high levels of poverty, humanitarianism and empathy were considered important components of a South African anaesthesiologist. Likewise, the ability to remain calm in situations of severe shortages accompanied by an overwhelming epidemic of trauma, was considered necessary. The multi-racial and diverse nature of South Africa's population also makes it essential that practitioners remain sensitive to patient differences and practise non-judgementally permission) in all their specialist roles. Humaneness is therefore considered a vital, core feature of a practitioner, and subsequently underpins all the roles of the anaesthesiologist.

Anaesthesiologists' non-technical skills are necessary for the practice of safe anaesthesia with good patient outcomes., ${ }^{6,19}$ Several suggestions from the panel alluded to the ability of the anaesthesiologist to be able to perform numerous technical and non-technical skills concurrently. These include situational awareness, not only in the collaborator role as indicated by CanMEDS, ${ }^{8}$ but in all roles of the specialist. The panel suggested that anaesthesiologists should be able to think on their feet and be creative in situations where traditional means of problem solving may not work or may be inappropriate due to resource limitations. Thinking outside the box and having the ability to be adaptable in any given context was deemed important. Thus the anaesthesiologist, in their quest for good patient outcomes, must learn to modify their practice in any situation, allowing context awareness to permeate across all their specialist roles.

Based on suggestions from the panel that were categorised into the two new meta-competencies of Humaneness and Context Awareness, the authors deemed it necessary to amalgamate these with the existing CanMEDS framework for local applicability. Humaneness, considered a central, personal role, is situated centrally at the core of the CanMEDS framework whilst context awareness is an all-encompassing role that infiltrates all others, embracing both personal and professional components of the anaesthesiologist (Figure 5).

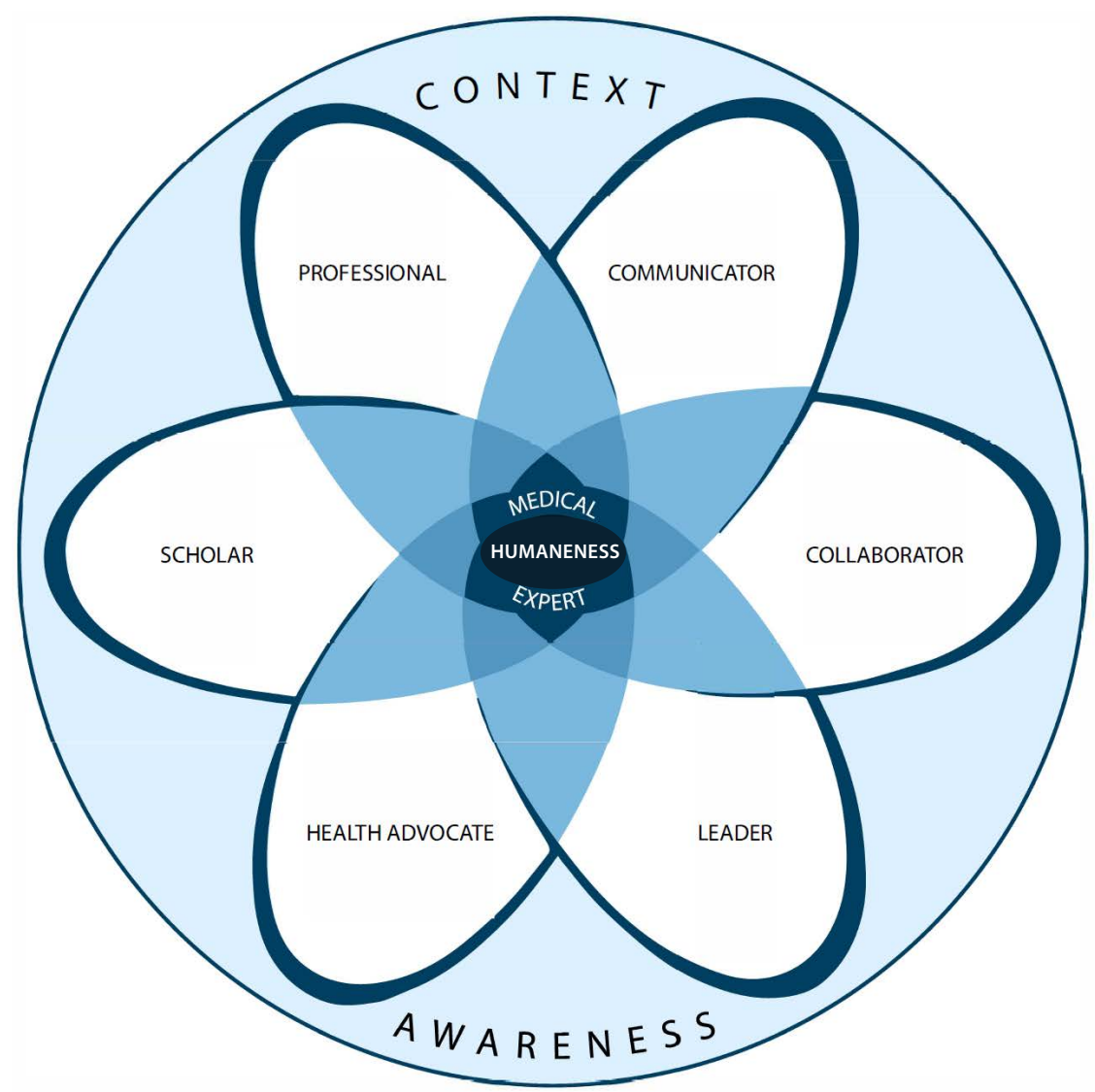

Figure 5. Modified CanMEDS for South African Anaesthesiology (Adapted from CanMEDS with 
This culminated in the creation of a comprehensive list of enabling competencies, specific to the South African anaesthesiology context, adapted from CanMEDS, which may be used for teaching and training and to assess FFP of local anaesthesiologists (Appendix).

Experts' weighting of the nine roles of the South African anaesthesiologist reflects that most roles should be evenly weighted with respect to time allocated for teaching, training and assessment. However, Medical Expert and Professional were weighted higher than the remaining seven meta-competencies. The large interquartile (IQ) ranges associated with Medical Expert and Professional indicate wider divergence of opinion despite the higher median scores. This alludes to the need for further research should these data be analysed for the purpose of curriculum development for South African anaesthesiology.

There were a number of limitations of this study, primarily related to the Delphi process. ${ }^{20-22}$ Relevant to this study, there are variable opinions as to how to define and determine consensus, ranging from $51 \%$ to $90 \% .{ }^{21,23}$ We determined that $80 \%$ would be appropriate for a high-stakes' study such as this. A larger sample size may be argued as a better representation of the South African anaesthesiology profession, however, limitations of panel size to ensure a successful Delphi process dictated the need for restrictive sampling. Our panel of 16, although slightly larger than the recommended number of 8-12 and logistically more difficult to manage, was however, representative of the entire country. Criticism exists regarding unequal distribution of expertise in panels, ${ }^{21}$ however this was not the case in our study. To follow up the potential lack of responses, investigators require respondent identities which may compromise the truthfulness of responses, ${ }^{22}$ however, in our study, participant responses were only known by a single investigator.

Our study had several strengths. The Delphi process was specifically chosen to generate consensus in a field where evidence is lacking. The sample is representative of all regions of South Africa and therefore attests to the generalised applicability nationally. Due to the absence of physical meetings, our expert panel was more geographically representative due to cost saving and less likely affected by peer pressure perceived from face-toface encounters..$^{24}$ Excellent response rates were achieved by the electronic process. This ensured that all opinions and judgements of experts in all rounds were considered and contributed to the validity of the data. ${ }^{23}$

\section{Conclusion}

This study provides some insight into the current perceptions of local anaesthesiology experts and has assisted in formulating a list of criteria against which South African anaesthesiology graduates can be measured to assess their fitness for purpose. Despite the current lack of local evidence in this regard, this study has succeeded in creating a modified CanMEDS framework that is applicable for use in all regions of South Africa. However, the question of whether South African anaesthesiology graduates are currently fit for purpose according to the modified framework, remains unanswered. Future research in this regard would require investigation of the various stakeholders' opinions. Results of such further inquiry will inform teaching, training and assessment and assist in optimisation of the current anaesthesiology curriculum to address any deficiencies that might exist.

\section{Acknowledgement}

The authors would like to thank Dr Santosh Pershad, Professor Reitze Rodseth and Mrs Shannon Whittle for their assistance.

Disclosure of Interest: The authors report no conflicts of interest ORCID

\section{N Kalafatis (iD) https://orcid.org/0000-0002-1675-1963} PD Gopalan (iD https://orcid.org/0000-0002-3816-1171

\section{References}

1. Beckett M, Hulbert D, Brown R. The new consultant survey 2005. Emerg Med J. 2006;23(6):461-3.

2. Dijkstra IS, Pols J, Remmelts P, Brand PLP. Preparedness for practice: a systematic cross-specialty evaluation of the alignment between postgraduate medical education and independent practice. Medical Teacher. 2015;37(2):153-61.

3. Westerman M, Teunissen PW, Fokkema JP, et al. The transition to hospital consultant and the influence of preparedness, social support, and perception: A structural equation modelling approach. Med Teach. 2013;35(4):320-7.

4. Westerman $M$, Teunissen $P W$, Jørgensen $R L$, et al. The transition to hospital consultant: Denmark and the Netherlands compared on preparedness for practice, perceived intensity and contextual factors. Medical Teacher. 2013;35(6):481-9.

5. Rama-Maceiras $P$, Jokinen J, Kranke $P$. Stress and burnout in anaesthesia: a real world problem? Current Opinion In Anaesthesiology. 2015;28(2):151-8.

6. Kalafatis NA Sommerville TE Gopalan PD. Fitness for purpose in anaesthesiologya review. Southern African Journal of Anaesthesia and Analgesia, 2018; 24(6) :148-154

7. Frank JR. The CanMEDS 2005 physician competency framework.Better standards Better physicians. Better care. Ottawa: The Royal College of Physicians and Surgeons of Canada. 2005.

8. Frank J, Snell L, Sherbino Jea, editors. The Draft CanMEDS 2015 Physician Competency Framework-Series IV.Ottawa: The Royal College of Physicians and Surgeons of Canada;2015 March. 2015.

9. Ringsted C, Hansen TL, Davis D, Scherpbier A. Are some of the challenging aspects of the CanMEDS roles valid outside Canada? Med Educ. 2006;40(8):807-15.

10. Ortwein $H$, Knigg $M$, Rehberg $B$, Vargas $H$, Spies $C$. Validation of core competencies during residency training in anaesthesiology. German Medical Science: GMS E-Journal. 2011;9.

11. Frank J, Snell L, Sherbino J. Draft CanMEDS 2015 Milestones Guide-September 2014. Ottawa: The Royal College of Physicians and Surgeons of Canada; 2014-September. 2014.

12. Leung W-C. Learning in Practice. BMJ. 2002;325:693-6.

13. Patel BS, Feerick $A$. Will competency assessment improve the training and skills of the trainee anaesthetist? Anaesthesia. 2002;57:710-1.

14. Snell LS, Frank JR. Competencies, the tea bag model, and the end of time. Med Teach. 2010;32(8):629-30.

15. Holmboe ES, Ward DS, Reznick RK, et al. Faculty development in assessment: the missing link in competency-based medical education. Acad Med 2011;86(4):460-7.

16. Berkenbosch L, Brouns JW, Heyligers I, Busari JO. How Dutch medical residents perceive their competency as manager in the revised postgraduate medical curriculum. Postgrad Med J. 2011;87(1032):680-7.

17. Verma S, Flynn L, Seguin R. Faculty's and residents' perceptions of teaching and evaluating the role of health advocate: a study at one Canadian university. Academic Medicine. 2005;80(1):103-8.

18. Dreyfus H, Dreyfus S. Mind over Machine: The Power of Human Intuition and Expertise in the Era of the Computer. New York: New York: The Free Press; 1986.

19. Patey R, Flin R, Fletcher G, Maran N, Glavin R. Developing a Taxonomy of Anesthetists' Nontechnical Skills (ANTS). 2005. In: Advances in Patient Safety: From research to Implementation (Vol 4: Programs,Tools and Products) [Internet]. Agency for Healthcare Research and quality (US); [325-36].

20. Powell C. The Delphi Technique:myths and realities. Journal of Advanced 2003;41(4):376-82.

21. Hsu C, Sandford B. The Delphi Technique: Making sense of Consensus. Practical assessment,Research \& Evaluation. 2007;12(10):1-8.

22. Thangaratinam S, Redman C. The Delphi Technique. The Obstetrician \& Gynaecologist. 2005;7:120-5.

23. Hasson F, Keeney S, McKenna H. Research guidelines for the Delphi survey technique. Journal of Advanced Nursing. 2000;32(4):1008-15.

24. Kearney RA. Defining professionalism in anaesthesiology. Medical Education. 2005;39(8):769-76. 


\section{Appendix}

MEDICAL EXPERT

\section{Key Competency:}

1. Practise medicine within their defined scope of practice and expertise

2. Perform a patient-centred clinical assessment and establish a management plan.

3. Plan and perform procedures and therapies for the purpose of assessment and/or management.

4. Establish plans for ongoing care and, when appropriate, timely consultation.

5. Actively contribute, as an individual and as a member of a team providing care, to the continuous improvement of health care quality and patient safety.

\section{COMMUNICATOR}

\section{Key Competency:}

1. Establish professional therapeutic relationships with patients and their families.

2. Elicit and synthesise accurate and relevant information, incorporating the perspectives of patients and their families.

3. Share health care information and plans with patients and their families.

4. Engage patients and their families in developing plans that reflect the patient's health care needs and goals.

5. Document and share written and electronic information about the medical encounter to optimize clinical decisionmaking, patient safety, confidentiality, and privacy.

\section{Enabling Competencies:}

1.1 Demonstrate a commitment to high-quality care of their patients

1.2 Integrate the CanMEDS Intrinsic Roles into their practice of medicine

1.3 Apply knowledge of the clinical and biomedical sciences relevant to their discipline

1.4 Perform appropriately timed clinical assessments with recommendations that are presented in an organized manner

1.5 Carry out professional duties in the face of multiple, competing demands

1.6 Recognize and respond to the complexity, uncertainty, and ambiguity inherent in medical practice

2.1 Prioritize issues to be addressed in a patient encounter

2.2 Elicit a history, perform a physical exam, select appropriate investigations, and interpret their results for the purpose of diagnosis and management, disease prevention, and health promotion

2.3 Establish goals of care in collaboration with patients and their families, which may include slowing disease progression, treating symptoms, achieving cure, improving function, and palliation

2.4 Establish a patient-centred management plan

3.1 Determine the most appropriate procedures or therapies

3.2 Obtain and document informed consent, explaining the risks and benefits of, and the rationale for, a proposed procedure or therapy

3.3 Prioritize a procedure or therapy, taking into account clinical urgency and available resources

3.4 Perform a procedure in a skillful and safe manner, adapting to unanticipated findings or changing clinical circumstances

4.1 Implement a patient-centred care plan that supports ongoing care, follow-up on investigations, response to treatment, and further consultation

5.1 Recognize and respond to harm from health care delivery, including patient safety incidents

5.2 Adopt strategies that promote patient safety and address human and system factors

5.3 Demonstrate the ability to multi-task

\section{Enabling Competencies:}

1.1 Communicate using a patient-centred approach that encourages patient trust and autonomy and is characterized by empathy, respect, and compassion

1.2 Optimize the physical environment for patient comfort, dignity, privacy, engagement, and safety

1.3 Recognize when the values, biases, or perspectives of patients, physicians, or other health care professionals may have an impact on the quality of care, and modify the approach to the patient accordingly

1.4 Respond to a patient's non-verbal behaviours to enhance communication

1.5 Manage disagreements and emotionally charged conversations

1.6 Adapt to the unique needs and preferences of each patient and to his or her clinical condition and circumstances

2.1 Use patient-centred interviewing skills to effectively gather relevant biomedical and psychosocial information

2.2 Provide a clear structure for and manage the flow of an entire patient encounter

2.3 Seek and synthesize relevant information from other sources, including the patient's family, with the patient's consent

3.1 Share information and explanations that are clear, accurate, and timely, while checking for patient and family understanding

3.2 Disclose harmful patient safety incidents to patients and their families accurately and appropriately

4.1 Facilitate discussions with patients and their families in a way that is respectful, non-judgmental, and culturally safe

4.2 Use communication skills and strategies that help patients and their families make informed decisions regarding their health

5.1 Document clinical encounters in an accurate, complete, timely, and accessible manner, in compliance with regulatory and legal requirements

5.2 Communicate effectively using a written health record, electronic medical record, or other digital technology

5.3 Share information with patients and others in a manner that respects patient privacy and confidentiality and enhances understanding

5.4 Able to communicate effectively with patients, care-givers and families despite language differences 


\section{Key Competency:}

1. Work effectively with physicians and other colleagues in the health care professions.

2. Work with physicians and other colleagues in the health care professions to promote understanding, manage differences, and resolve conflicts.

3. Hand over the care of a patient to another health care professional to facilitate continuity of safe patient care.

LEADER

\section{Key Competency:}

1. Contribute to the improvement of health care delivery in teams, organisations, and systems

2. Engage in the stewardship of health care resources.

\section{Demonstrate leadership in} professional practice.

4. Manage career planning, finances, and health human resources in a practice.

\section{HEALTH ADVOCATE}

\section{Key Competency:}

1. Respond to an individual patient's health needs by advocating with the patient within and beyond the clinical environment

\section{Respond to the needs of the} communities or populations they serve by advocating with them for systemlevel change in a socially accountable manner

SCHOLAR

\section{Enabling Competencies:}

1.1 Establish and maintain positive relationships with physicians and other colleagues in the health care professions to support relationship-centred collaborative care

1.2 Negotiate overlapping and shared responsibilities with physicians and other colleagues in the health care professions in episodic and ongoing care

1.3 Engage in respectful shared decision-making with physicians and other colleagues in the health care professions

1.4 Ability to respond to need for emergency involvement outside perioperative domain

2.1 Show respect toward collaborators

2.2 Implement strategies to promote understanding, manage differences, and resolve conflicts in a manner that supports a collaborative culture

3.1 Determine when care should be transferred to another physician or health care professional

3.2 Demonstrate safe handover of care, using both verbal and written communication, during a patient transition to a different health care professional, setting, or stage of care

3.3 Awareness of the competency required of the receiving practitioner at hand over of patients

\section{Key Competency:}

1. Engage in the continuous enhancement of their professional activities through ongoing learning

2. Teach students, residents (trainees), the public, and other health care professionals

3. Integrate best available evidence into practice.

4. Contribute to the creation and dissemination of knowledge and practices applicable to health.

\section{Enabling Competencies:}

1.1 Apply the science of quality improvement to contribute to improving systems of patient care

1.2 Contribute to a culture that promotes patient safety

1.3 Analyze patient safety incidents to enhance systems of care

1.4 Use health informatics to improve the quality of patient care and optimize patient safety

2.1 Allocate health care resources for optimal patient care

2.2 Apply evidence and management processes to achieve cost-appropriate care

3.1 Demonstrate leadership skills to enhance health care

3.2 Facilitate change in health care to enhance services and outcomes

4.1 Set priorities and manage time to integrate practice and personal life

4.2 Manage a career and a practice

4.3 Implement processes to ensure personal practice improvement

\section{Enabling Competencies:}

1.1 Incorporate disease prevention, health promotion, and health surveillance into interactions with individual patients

2.1 Possess an In-depth knowledge of global health issues

2.2 Improve clinical practice by applying a process of continuous quality improvement to disease prevention, health promotion, and health surveillance activities

2.3 Contribute to a process to improve health in the community or population they serve

2.4 Ensure that all staff (including oneself) in the clinical environment are safe and not at risk with respect to physical or psychological injuries.

\section{Enabling Competencies:}

1.1 Develop, implement, monitor, and revise a personal learning plan to enhance professional practice

1.2 Identify opportunities for learning and improvement by regularly reflecting on and assessing their performance using various internal and external data sources

1.3 Engage in collaborative learning to continuously improve personal practice and contribute to collective improvements in practice.

2.1 Recognize the influence of role-modelling and the impact of the formal, informal, and hidden curriculum on learners

2.2 Promote a safe learning environment

2.3 Ensure patient safety is maintained when learners are involved

2.4 Plan and deliver a learning activity

2.5 Provide feedback to enhance learning and performance

2.6 Assess and evaluate learners, teachers, and programs in an educationally appropriate manner

3.1 Recognize practice uncertainty and knowledge gaps in clinical and other professional encounters and generate focused questions that address them

3.2 Identify, select, and navigate pre-appraised resources

3.3 Critically evaluate the integrity, reliability, and applicability of health-related research and literature

3.4 Integrate evidence into decision-making in their practice

4.1 Demonstrate an understanding of the scientific principles of research and scholarly inquiry and the role of research evidence in health care

4.2 Identify ethical principles for research and incorporate them into obtaining informed consent, considering potential harms and benefits, and considering vulnerable populations

4.3 Summarize and communicate to professional and lay audiences, including patients and their families, the findings of relevant research and scholarly inquiry 


\section{Key Competency:}

1. Demonstrate a commitment to patients by applying best practices and adhering to high ethical standards

\section{Enabling Competencies:}

1.1 Exhibit appropriate professional behaviours and relationships in all aspects of practice, demonstrating honesty, integrity, humility, commitment, compassion, respect, altruism, respect for diversity, and maintenance of confidentiality

1.2 Demonstrate a commitment to excellence in all aspects of practice

1.3 Recognize and respond to ethical issues encountered in practice

1.4 Recognize and manage conflicts of interest

1.5 Exhibit professional behaviours in the use of technology-enabled communication

2. Demonstrate a commitment to society by recognizing and responding to societal expectations in health care.

2.1 Demonstrate accountability to patients, society, and the profession by responding to societal expectations of physicians

2.2 Demonstrate a commitment to patient safety and quality improvement

\section{Demonstrate a commitment to the} profession by adhering to standards and participating in physician-led regulation.

4. Demonstrate a commitment to physician health and well-being to foster optimal patient care.
3.1 Fulfill and adhere to the professional and ethical codes, standards of practice, and laws governing practice

3.2 Recognize and respond to unprofessional and unethical behaviours in physicians and other colleagues in the health care professions

4.1 Exhibit self-awareness and manage influences on personal well-being and professional performance

4.2 Manage personal and professional demands for a sustainable practice throughout the physician life cycle

4.3 Promote a culture that recognizes, supports, and responds effectively to colleagues in need

4.4 Able to practice efficiently at all times

4.5 Ability to advocate on behalf of the profession to the public to assist in public awareness.

4.6 Able to function punctually at all times

CONTEXT AWARENESS

\section{Key Competency:}

1. Demonstrate the ability to adapt clinical and non-clinical practice to specific scenarios and environments to ensure good outcomes.

\section{Enabling Competencies:}

1.1 Ability to perceive, to comprehend the meaning of, and to predict the impact of variables in the work environment (situational awareness)

1.2 Capacity to be adaptable and creative in solving problems

1.3 Ability to assess capacity and obtain appropriate consent in any given circumstance

1.4 Ability to remain humane in difficult circumstances

\section{HUMANENESS}

\section{Key Competency:}

1. Demonstrate the ability to engage with and respond appropriately to patients, colleagues, societies and communities in need with qualities that personify good naturedness and with sound moral standards.

\section{Enabling Competencies:}

1.1 Awareness of and respect for the impact and sensitivities of race, culture and ethnicity on decisionmaking and patient management

1.2 Tolerance and the ability to respect diversities in patients, families and colleagues and function in a non-judgmental manner

1.3 Ability to practice with sound moral standards (Honesty, altruism, integrity, humility, commitment, kindness)

1.4 Ability to remain calm under pressure

1.5 Ability to manage patients and their families with a caring and empathetic nature.

1.6 Ability to promote human welfare and humanitarianism in society and at work 